\title{
ANÁLISIS DE MARCADORES MORFOLÓGICOS Y MOLECULARES EN PAPA (Solanum tuberosum L.)
}

\author{
ANALYSIS OF MORPHOLOGICAL AND MOLECULAR MARKERS IN POTATO \\ (Solanum tuberosum L.)
}

\author{
Rose Onamu, Juan P. Legaria Solano*, Jaime Sahagún Castellanos, José L. Rodríguez de la O y Joel Pérez Nieto
}

\begin{abstract}
Instituto de Horticultura, Departamento de Fitotecnia, Universidad Autónoma Chapingo. Km 38.5 Carr. México-Texcoco. 56230, Chapingo, Estado de México. Tel. 01(595)9521500.
\end{abstract}

*Autor para correspondencia (legarias.juan@yahoo.com)

\section{RESUMEN}

La información sobre identificación, diversidad genética y relaciones entre genotipos de papa (Solanum tuberosum L.) es de importancia para su conservación eficiente, mejoramiento genético y utilización de los recursos genéticos de esta especie. Por tanto, con el objetivo de evaluar la eficiencia de los caracteres morfológicos y marcadores tipo RAPD e ISSR para discriminar genotipos de papa, se caracterizaron 15 variedades de papa cultivadas en México. Se evaluaron 12 caracteres cuantitativos y tres cualitativos transformados a una escala nominal. Ambos marcadores detectaron diversidad genética entre variedades y las diferenciaron, pero la prueba de Mantel mostró baja correlación entre la matriz de similitud morfológica y la molecular. Los marcadores moleculares discriminaron completamente a los 15 cultivares analizados, mientras que los marcadores morfológicos fueron incapaces de discriminar algunos de ellos. Para que los programas de mejoramiento sean eficientes es importante seleccionar líneas con base en su diversidad genética y parámetros agro-morfológicos, asistidos con marcadores moleculares.

Palabras clave: Solanum tuberosum, caracterización, marcadores morfológicos, marcadores moleculares.

\section{SUMMARY}

Information about identification, genetic diversity and relationships between potato (Solanum tuberosum L.) genotypes is important for conservation, breeding programs and utilization of genetic resources of this species. With the objective of evaluating the efficiency of morphological characters and RAPD and ISSR markers to discriminate potato genotypes, 15 potato cultivars grown in México were characterized. Morphological characters included both 12 quantitative and three qualitative characters which were scored based on a nominal scale. Both markers demonstrated genetic diversity among the potato cultivars and differentiated between them. However, the Mantel test showed low correlation between the morphological similarity matrix and the molecular one. Molecular markers completely discriminated between all 15 cultivars, while morphological markers were unable to discriminate among some cultivars. In order to improve efficiency of the genetic breeding programs, it is important to select lines based on genetic diversity and agro-morphological parameters, assisted with molecular markers.

Index words: Solanum tuberosum, characterization, morphological markers, molecular markers.

\section{INTRODUCCIÓN}

La papa (Solanum tuberosum L.) es uno de los cultivos mas importantes a nivel mundial. Ocupa el cuarto lugar en producción para consumo humano, superado solamente por trigo (Triticum aestivum L.), arroz (Oryza sativa L.) y maíz (Zea mays L.). En México la papa se cultiva anualmente en una superficie de 69054 ha, con una producción de $1433239 \mathrm{t}$ y un rendimiento promedio de $26 \mathrm{t} \mathrm{ha}^{-1}$. Se cultiva principalmente en los Estados de Sinaloa, Sonora, Chihuahua, Coahuila, Guanajuato, Nuevo León, Jalisco, Michoacán, Veracruz y México (SAGARPA, 2011).

Resultados morfológicos y moleculares recientes clasifican a la papa en 190 especies repartidas en cuatro clados, que presentan algunas relaciones inter-específicas y gran variabilidad con la clasificación propuesta inicialmente en 1990 por Hawkes (Spooner et al., 2008). Spooner et al. (2007) ponen en tela de juicio muchas de estas series y enlistan únicamente 189 especies, a pesar de la descripción de diez nuevas especies desde Hawkes (1990), y predicen que en un futuro se presentará una nueva reducción en el número de especies, en la medida que continúen los trabajos de investigación con marcadores moleculares.

La taxonomía de las especies silvestres y cultivadas de papa continúa siendo notablemente compleja (Hijmans et al., 2003; Spooner et al., 2003; Spooner et al., 2004; Spooner et al., 2007; Spooner et al., 2008), ha generado controversias y actualmente sigue siendo refinada (Sponner et al., 2007). Muchas especies de papa son iguales, aunque tengan apariencia diferente, ya que mantienen la capacidad de hibridarse cuando están en contacto. El límite entre especies es confuso y aún falta definición para entenderlo claramente (Spooner y Lara-Cabrera, 2001; Lara Cabrera y Spooner, 2004). 
La información sobre identificación, diversidad genética y relaciones entre genotipos de papa es de importancia para la conservación eficiente, programas de mejoramiento $y$ utilización de los recursos genéticos. México es un país rico en diversidad genética de esta especie; sin embargo, estos recursos genéticos no se han aprovechado adecuadamente debido a la limitada información disponible sobre sus características y su diversidad genética (Becerra y Paredes, 2000).

La caracterización morfológica es el primer paso en el mejoramiento de los cultivos y programas de conservación. Las características morfológicas se han venido utilizando para estudiar la diversidad genética, identificar plantas cultivadas y conservar los recursos genéticos. A la fecha, los marcadores morfológicos establecen las bases para identificar y diferenciar variedades, pero presentan las siguientes limitantes: su obtención es tardada, son métodos complejos, limitados, subjetivos, influenciados por el ambiente, caros e involucran estados de desarrollo específicos del cultivo (Yasmin et al., 2006).

Se ha reportado diversidad genética entre genotipos de papa mediante el uso de marcadores morfológicos (Ahmadizadeh y Felenji, 2011; Solís et al., 2007; Arslanoglu et al., 2011). Chimote et al. (2007) reportaron la diversidad genética y relaciones entre genotipos de papa de la India, y con base en ella seleccionaron progenitores superiores.

Los marcadores moleculares, que han revolucionado la forma de conducir los estudios sobre diversidad genética, incluyen aquellos basados en la PCR (reacción en cadena de la polimerasa, por sus siglas en inglés) que son rápidos, objetivos, simples y abundantes, no afectados por el ambiente y pueden analizarse en cualquier etapa de desarrollo del cultivo. Los marcadores tipo RAPD e ISSR son simples, económicos, rápidos y discriminan eficientemente entre genotipos de papa (Orona-Castro et al., 2006; Rocha et al., 2010; Yasmin et al., 2006). Algunas de sus limitantes incluyen su naturaleza dominante y su inversión inicial y soporte técnico especializado. Con base a estos antecedentes, en el presente estudio se evaluó la eficiencia de los caracteres morfológicos y marcadores tipo RAPD e ISSR para discriminar genotipos de variedades de papa que se siembran en México.

\section{MATERIALES Y MÉTODOS}

\section{Germoplasma y caracterización morfológica}

Quince genotipos de papa (Cuadro 1) se evaluaron bajo condiciones controladas de invernadero en Chapingo, Estado de México ubicado a $2240 \mathrm{~m}$ de altitud, a $19^{\circ} 29^{\prime} \mathrm{LN}$ y $98^{\circ} 53^{\prime} \mathrm{LO}$. Tres tubérculos de cada cultivar se sembraron en macetas de $20 \mathrm{~cm}$ de diámetro. Se utilizaron cuatro repeticiones bajo un diseño completamente al azar, para un total de 12 x 15 = 60 plantas. En 12 individuos de cada cultivar se midieron 15 caracteres morfológicos (Cuadro 2), de acuerdo con la guía de descriptores varietales de la UPOV (2004). La fertilización y control de plagas se hizo de acuerdo con las recomendaciones técnicas para el cultivo de la papa en la región (INIA, 1981).

Cuadro 1. Lista de cultivares, su origen y tipo utilizados en el presente estudio.

\begin{tabular}{lllll}
\hline Abreviatura & \multicolumn{1}{c}{ Genotipo } & \multicolumn{1}{c}{ Origen } & \multicolumn{1}{c}{ Tipo } & \multicolumn{1}{c}{ Genealogía } \\
\hline CRM & 'Cambray Rosa Morelos' & México & Variedad criolla & Desconocida \\
CE & 'Criolla Edo Méx' & México & Variedad criolla & Desconocida \\
AGE & 'Alfa' & Europa & Cultivar mejorado & Paul Kruger x Preferent \\
ATL & 'Atlantic' & U.S.A & Cultivar mejorado & Waseon x B5141-6 \\
ARM & 'Armada' & Europa & Cultivar mejorado & Rianta x AR 76 16 81 \\
FIO & 'Fianna' & Europa & Cultivar mejorado & KO62-660 x AM-66-42 \\
MOTE & 'Montserrat' & México & Cultivar mejorado & 7-AM-9 x 57-DZ-23 \\
MDL & 'Mondial' & Europa & Cultivar mejorado & Spunta x SVP Ve 66295 \\
GIG & 'Gigant' & Europa & Cultivar mejorado & Elvira x AM-66-42 \\
CBLANC & 'Cambray Blanca Edo Méx' & México & Variedad criolla & Desconocida \\
TOLL & 'Tollocan' & México & Cultivar mejorado & 58-ES-37 x 58-ER-1 \\
MOCH & 'Mochis' & México & Cultivar mejorado & Desconocida \\
CAMB RD & 'Cambray Rosa DF' & México & Variedad criolla & Desconocida \\
CRIO BP & 'Criolla Blanca Puebla' & México & Variedad criolla & Desconocida \\
PAPA CHIC & 'Papa Chica' & México & Variedad criolla & Desconocida \\
\hline
\end{tabular}


Cuadro 2. Caracteres morfológicos cuantitativos ( 1 a 12) y cualitativos (13 a 15) evaluados en variedades de papa.

\begin{tabular}{|c|c|c|c|c|c|c|}
\hline \multirow{2}{*}{\multicolumn{2}{|c|}{ Carácter }} & \multicolumn{4}{|c|}{ Valor $7 / 5 / 3 / 1$} & \\
\hline & & 7 & 5 & 3 & 1 & \\
\hline 1 & Altura de planta & Alta & Intermedia & Baja & & \\
\hline 2 & Hábito de crecimiento & Extendido & Semi-extendido & Erecto & & \\
\hline 3 & Intensidad de pigmentación del tallo & Fuerte & Moderada & Débil & Verde & \\
\hline 4 & Silueta de la hoja & Abierta & Intermedia & Cerrada & & \\
\hline 5 & Ancho de las hojas & Ancha & Mediana & Angosta & & \\
\hline 6 & Coalescencia de las hojas & Alta & Moderada & Baja & & \\
\hline 7 & Madurez del tubérculo & Tardía & Intermedia & Temprana & & \\
\hline 8 & Hojas secundarias & Altas & Moderadas & Pocas & Ausentes & \\
\hline 9 & Tamaño de hojas & Grandes & Medianas & Pequeñas & & \\
\hline 10 & Longitud de hojuelas-ve & Largas & Medianas & Cortas & & \\
\hline 11 & Longitud de hojuelas +ve & Largas & Medianas & Cortas & & \\
\hline \multirow[t]{3}{*}{12} & Ancho de la hoja terminal & Ancha & Mediana & Angosta & & \\
\hline & & \multicolumn{5}{|c|}{ Valor 5/4/3/2/1 } \\
\hline & & 5 & 4 & 3 & 2 & 1 \\
\hline 13 & Tipo de planta & & & Frondosa & Intermedia & Tipo copa \\
\hline 14 & Forma del tubérculo & & & Oblongo & Ovalado & Redondo \\
\hline 15 & Color de cubierta del tubérculo & & & & Roja & Blanca \\
\hline
\end{tabular}

\section{Caracterización molecular}

La purificación del ADN se hizo de acuerdo con Dellaporta et al. (1983), con algunas modificaciones. Secciones de $0.3 \mathrm{~g}$ de cinco tubérculos por genotipo se maceraron en nitrógeno líquido hasta obtener un polvo muy fino. Cada muestra se colocó en un microtubo (eppendorf) de $1.5 \mathrm{~mL}$ que contenía $600 \mu \mathrm{L}$ de amortiguador de extracción $(20 \mathrm{~mL}$ Tris-HCL $1 \mathrm{M}, \mathrm{pH}$ 8.0; $20 \mathrm{~mL}$ EDTA $0.5 \mathrm{M}, \mathrm{pH}$ 8.0; $20 \mathrm{~mL}$ $\mathrm{NaCl} 5 \mathrm{M} ; 35 \mu \mathrm{L} \beta$-mercaptoetanol; $40 \mathrm{~mL}$ dodecil sulfato de sodio $20 \%$ ) y se incubó a $65^{\circ} \mathrm{C}$ por $10 \mathrm{~min}$, con inversión ocasional de los tubos. Después se adicionó $200 \mu \mathrm{L}$ de acetato de potasio $5 \mathrm{M}$, se mezcló por inversión y se incubó en hielo por $30 \mathrm{~min}$. Se centrifugó a $8000 \times \mathrm{g}$ durante 10 min a temperatura ambiente, y el sobrenadante se transfirió a otro tubo que contenía $700 \mu \mathrm{L}$ de isopropanol frío (-20 ${ }^{\circ} \mathrm{C}$ ). Se mezcló por inversión e incubó a $-20^{\circ} \mathrm{C}$ por $30 \mathrm{~min}$ y centrifugó por $5 \mathrm{~min}$ a $8000 \times g$ a temperatura ambiente. El sobrenadante se eliminó, se recobró el precipitado y se disolvió en $200 \mu \mathrm{L}$ de solución para diluir (Tris-HCl $50 \mathrm{mM}$, EDTA- $\mathrm{Na}_{2} 10 \mathrm{mM}, \mathrm{pH} 8.0$ ).

Para eliminar el ARN se añadió $2 \mu \mathrm{L}$ de ARNasa A y se incubó a $37^{\circ} \mathrm{C}$ por $1 \mathrm{~h}$. Después se adicionó $20 \mu \mathrm{L}$ de acetato de sodio $3 \mathrm{M}$ más $200 \mu \mathrm{L}$ de isopropanol, se mezcló por inversión y se dejó precipitar a $-20^{\circ} \mathrm{C}$ por $2 \mathrm{~h}$. Se centrifugó a $8000 \times g$ por 5 min a temperatura ambiente. El sobrenadante se eliminó y el precipitado se lavó con $300 \mu \mathrm{L}$ de etanol 70 $\%$. La pastilla se secó y se disolvió en amortiguador TE (Tris$\mathrm{HCl} 10 \mathrm{mM}$, EDTA-Na $21 \mathrm{mM}, \mathrm{pH} 8.0)$ a $4{ }^{\circ} \mathrm{C}$. La concen- tración del ADN se cuantificó con un espectrofotómetro Genesys 10 UV Scanning ${ }^{\circledR}$ (Thermo Scientific; USA) y la calidad se verificó mediante electroforesis en un gel de agarosa $0.8 \%(\mathrm{p} / \mathrm{v})$. El ADN obtenido se usó en subsecuentes reacciones de PCR a una concentración de $10 \mathrm{ng} \mu \mathrm{L}^{-1}$.

\section{Condiciones de reacción para marcadores moleculares tipo RAPD}

Se ensayaron 25 iniciadores RAPD de las series A, B y D de Operon ${ }^{\circledR}$ (Operon Technologies Inc.; Alameda, CA, USA) de los cuales se seleccionaron cinco que mostraron polimorfismo y patrones de bandas claras y nítidas (Cuadro 3). Las reacciones de PCR se hicieron en un termociclador Techne ${ }^{\circledR}$ (TC-512; United Kingdom). La mezcla de reacción se llevó a cabo en un volumen de $25 \mu \mathrm{L}$ que contenía $4.2 \mu \mathrm{L}$ agua bi-destilada estéril, $10 \mu \mathrm{L}$ de dNTPs $(500 \mu \mathrm{M})$, $2.5 \mu \mathrm{L}$ de amortiguador 10X (Tris- $\mathrm{HCl} 750 \mathrm{mM}, \mathrm{pH} 8.8$; $\left(\mathrm{NH}_{4}\right)_{2} \mathrm{SO}_{4} 200 \mathrm{mM}$; Tween 20 a $\left.1 \%(\mathrm{v} / \mathrm{v})\right) ; 1.0 \mu \mathrm{L}$ de $\mathrm{MgCl}_{2}$ $(50 \mathrm{mM}) ; 3.0 \mu \mathrm{L}$ de iniciador a una concentración de 10 pM; $0.3 \mu \mathrm{L}$ de enzima Taq ADN polimerasa (Fermentas Life

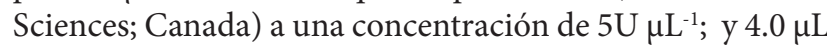
de $\mathrm{ADN}$ genómico a una concentración de $10 \mathrm{ng} \mu \mathrm{L}^{-1}$. Las condiciones de reacción fueron: un ciclo a $94^{\circ} \mathrm{C}, 2 \mathrm{~min} ; 38$ ciclos $\left[94^{\circ} \mathrm{C}\right.$ por $30 \mathrm{~s} ; 40^{\circ} \mathrm{C}$ por $30 \mathrm{~s} ; 72^{\circ} \mathrm{C}$ por $\left.90 \mathrm{~s}\right]$, con una extensión de $72{ }^{\circ} \mathrm{C}$ por $2 \mathrm{~min}$. Los fragmentos amplificados se separaron por electroforesis en un gel de agarosa a $1.2 \%(\mathrm{p} / \mathrm{v})$ con amortiguador TAE (40 mM Tris-acetato, pH 7.6; $1 \mathrm{mM} \mathrm{Na}_{2}$ EDTA), por $1 \mathrm{~h}$ a $120 \mathrm{~V}$. Los geles se tiñeron en bromuro de etidio $\left(0.5 \mathrm{mg} \mathrm{mL}^{-1}\right)$ por $15 \mathrm{~min}$; el 
exceso de colorante se eliminó por lavado del gel con agua destilada durante 5 min y se documentó bajo luz UV. Las reacciones de amplificación se repitieron al menos dos veces con el fin de verificar la repetitividad de los resultados.

\section{Condiciones de reacción para marcadores moleculares tipo ISSR}

Se usaron 5 iniciadores de un total de 10 ensayados (Cuadro 3). Las reacciones de PCR se realizaron en un termociclador Techne ${ }^{\circledR}$ (TC-512; United Kingdom). La mezcla de reacción se efectuó en $25 \mu \mathrm{L}$ que contenían $4.2 \mu \mathrm{L}$ de agua bi-destilada estéril, $10 \mu \mathrm{L}$ de dNTPs $(500 \mu \mathrm{M}), 2.5 \mu \mathrm{L}$ de amortiguador 10X (Tris- $\mathrm{HCl} 750 \mathrm{mM}, \mathrm{pH} 8.8 ;\left(\mathrm{NH}_{4}\right)_{2} \mathrm{~S}_{4}$ $200 \mathrm{mM}$; Tween $201 \%(\mathrm{v} / \mathrm{v})$ ); $1.0 \mu \mathrm{L}$ de $\mathrm{MgCl}_{2}(50 \mathrm{mM})$; $3.0 \mu \mathrm{L}$ de iniciador a una concentración de $10 \mathrm{pM} ; 0.3 \mu \mathrm{L}$ de enzima Taq ADN polimerasa (Fermentas Life Sciences; Canada) a una concentración de $5 \mathrm{U}_{\mu \mathrm{L}^{-1}}$ y $4.0 \mu \mathrm{L}$ de $\mathrm{ADN}$ genómico a una concentración de $10 \mathrm{ng} \mu \mathrm{L}^{-1}$.

Las condiciones de reacción fueron: $94{ }^{\circ} \mathrm{C}$ por $5 \mathrm{~min}, 30$ ciclos a $94{ }^{\circ} \mathrm{C}$ por $30 \mathrm{~s}$, temperatura especifica de alineamiento por $45 \mathrm{~s}$ más $72{ }^{\circ} \mathrm{C}$ por $2 \mathrm{~min}$, y un ciclo de extensión final a $72{ }^{\circ} \mathrm{C}$ por $10 \mathrm{~min}$. La temperatura de alineamiento estuvo en un rango de 40 a $58^{\circ} \mathrm{C}$, como se indica en el Cuadro 3 para cada iniciador, y los ciclos se redujeron a 30. Los fragmentos amplificados se separaron por electroforesis en geles de agarosa $1.5 \%(\mathrm{p} / \mathrm{v})$ con amortiguador TAE (40 mM Tris-acetato, $\mathrm{pH} 7.6 ; 1 \mathrm{mM} \mathrm{Na}_{2}$ EDTA), por $1 \mathrm{~h}$ a $120 \mathrm{~V}$. Los geles se tiñeron con bromuro de etidio $(0.5$ $\mathrm{mg} \mathrm{mL}^{-1}$ ) por 15 min y el exceso de colorante se eliminó por enjuague en agua destilada por 15 min y documentados bajo luz UV. Las reacciones de amplificación se repitieron al menos dos veces con el fin de verificar la repetitividad de los resultados.

\section{Análisis estadístico}

Los datos morfológicos (12 variables cuantitativas y tres variables cualitativas en escala nominal, en los que previamente se definieron tres o cuatro estados en cada caso, como se indica en el Cuadro 2) se analizaron construyendo primero una matriz básica de datos (MBD) para luego hacer un análisis de conglomerados mediante el coeficiente SM (Sneath y Sokal, 1973) y el programa NTSYS-pc versión 2.1 (Rohlf, 2000).

Se obtuvieron dendrogramas de relaciones entre las accesiones con el método UPGMA (Unweighted Pair Group Method using Arithmetic Averages) aplicado a datos de variables cuantitativas y cualitativas por separado y datos consenso de los dos tipos de marcadores (datos de variables cuantitativas más cualitativas). A los datos moleculares se les asignó un valor de uno (1) a la presencia de una banda y de cero (0) a la ausencia de la misma. Se evaluó un total de 146 loci, 64 de RAPD y 82 de ISSR (Cuadro 3), y con ellos se construyó una matriz básica de datos (MBD). Para el análisis de estos datos también se usó el coeficiente de concordancia simple (SM) (Sneath y Sokal, 1973) y el programa NTSYS-pc versión 2.1 (Rohlf, 2000).

Luego se obtuvo un dendrograma de relaciones entre las accesiones con el método UPGMA con los datos de RAPD e ISSR por separado y datos consenso de los dos tipos de marcadores (datos de RAPDs más ISSR). Se hizo una prueba de Mantel (Mantel, 1967) para obtener correlaciones entre matrices de similitud, con el programa Mantel Nonparametric Test calculator para Windows Versión 2.0 (1999-2007). Finalmente, el escalado multidimensional no métrico se hizo mediante el programa NTSYS-pc versión 2.1 (Rohlf, 2000). El propósito de este análisis fue de contrastar los agrupamientos generados por este método contra los de conglomerados.

Cuadro 3. Lista de iniciadores RAPD e ISSR, secuencias 5' -3', temperatura de alineamiento (TA), productos amplificados (PA) y polimorfismo detectado.

\begin{tabular}{llllc}
\hline Iniciador & Secuencia (5'-3') & TA & PA & Polimorfismo (\%) \\
A02 & TGCCGAGCTG & 40 & 14 & 77.70 \\
A05 & AGGGGTCTTG & 40 & 12 & 92.30 \\
D10 & GGTCTACACC & 40 & 17 & 90.90 \\
D11 & AGCGCCATTG & 40 & 11 & 94.40 \\
D02 & GGACCCAACC & 40 & 10 & 87.50 \\
P1 & GAGCAACAACAACAACAA & 48 & 14 & 57.10 \\
P2 & CTGAGAGAGAGAGAGAGAG & 58 & 18 & 80.00 \\
P3 & AGAGAGAGAGAGAGAGTG & 45 & 21 & 92.80 \\
P4 & ATGATGATGATGATG & 40 & 14 & 78.60 \\
P5 & AGAGAGAGAGAGAGAG & 45 & 15 & 92.80 \\
\hline
\end{tabular}




\section{RESULTADOS Y DISCUSIÓN}

\section{Caracterización morfológica y molecular mediante análisis de conglomerados}

Los caracteres cuantitativos diferenciaron a los 15 cultivares en tres grupos con un coeficiente de 0.47 (Figura 1A), dentro de un rango de 0.00 a 1.00. El primer grupo consistió de 'Cambray Rosa Morelos' y 'Cambray Rosa DF', dos cultivares criollos que formaron el grupo más divergente. El segundo grupo incluyó a 'Papa Chica,' 'Mondial,' 'Fianna', 'Atlantic,', 'Mochis', 'Montserrat' y 'Criolla Edo Méx. El tercer grupo consistió de 'Alfa' 'Tollocan', 'Criolla Blanca Puebla', 'Armada', 'Gigant' y 'Cambray Blanca Edo Méx'. La más alta similitud (1.00) se observó entre 'Cambray Rosa Morelos' y 'Cambray Rosa DF, 'Criolla Edo Méx’ y 'Mochis,' 'Criolla Edo Méx' y 'Montserrat'. Algunos cultivares que mostraron similitudes más bajas (cercanas a 0.00) fueron 'Cambray Blanca Edo Méx' y 'Mochis', 'Montserrat', 'Atlantic', 'Fianna' y 'Cambray Rosa Morelos.' 'Fianna' y 'Armada' fueron los cultivares que mostraron los valores más bajos de similitud $(0.00)$ en caracteres cuantitativos.

Los caracteres cualitativos discriminaron a los 15 cultivares en tres grupos a un coeficiente de 0.41 (Figura 1B); los coeficientes de similitud variaron entre 0.00 y 0.89 . El primer grupo consistió de 'Cambray Rosa Morelos', 'Cambray Rosa DF' y 'Papa Chica'. Este grupo fue más divergente que el resto de cultivares. El segundo grupo lo formaron 'Cambray Blanca Edo Méx' y 'Mochis.' El tercer grupo consistió de 'Alfa,' 'Armada,' 'Fianna,' 'Atlantic', 'Mondial,' 'Criolla Blanca Puebla, 'Gigant,' 'Montserrat', 'Tollocan' y 'Criolla Edo Méx'. Una alta similitud (0.89) se registró entre 'Armada' y 'Fianna' y 'Montserrat' y 'Tollocan. 'Cambray Rosa Morelos' y 'Armada’ fueron los más distantes (0.00).

Un análisis combinado de caracteres cualitativos y cuantitativos permitió la formación de cuatro grupos a un coeficiente de 0.45 (Figura 2), en un rango de similitud entre 0.067 a 0.87 . El primer grupo consistió de 'Cambray Rosa Morelos' y 'Cambray Rosa DF', que también fue el grupo más divergente. 'Papa Chica' formó el segundo grupo. El tercero agrupó a 'Alfa, 'Armada' y 'Cambray Blanca Edo Méx. El cuarto grupo se integró con 'Fianna', 'Mochis', 'Atlantic', 'Mondial,' 'Criolla Edo Méx', 'Montserrat', 'Tollocan', 'Gigant' y 'Criolla Blanca Puebla'. Alta similitud se detectó entre 'Cambray Rosa Morelos' y 'Cambray Rosa DF, 'Montserrat' y 'Tollocan', 'Armada' y 'Gigant,' 'Gigant' y 'Criolla Blanca Puebla,' 'Tollocan' y 'Criolla Blanca Puebla'. Los menores coeficientes de similitud se obtuvieron para 'Cambray Rosa Morelos' y 'Armada', 'Cambray Rosa Morelos' y 'Alfa', 'Cambray Blanca Edo Méx' y 'Criolla Edo Méx', ‘Cambray Blanca Edo Méx’ y ‘Cambray Rosa Morelos'.
El análisis molecular con datos consenso de RAPD e ISSR discriminó a los 15 cultivares en seis grupos a un coeficiente de 0.77 (Figura 3), con similitudes en un rango de 0.61 a 0.94. 'Cambray Rosa Morelos' aglutinó el primer grupo, que también fue el más alejado del resto. 'Mondial' conformó el segundo grupo. El tercer grupo consistió de 'Montserrat' y 'Tollocan', dos variedades mexicanas. 'Criolla Edo Méx' y 'Papa Chica' formaron el cuarto grupo. 'Gigant' formó el quinto. El sexto grupo consistió de cultivares mexicanos ('Mochis', 'Cambray Blanca Edo Méx', 'Cambray Rosa DF' y 'Criolla Blanca Puebla'), 'Atlantic' de Norteamérica y cultivares europeos ('Alfa', 'Armada' y 'Fianna'). Alta similitud se observó entre 'Fianna' y 'Armada' (0.90), 'Alfa' y 'Armada', 'Cambray Blanca Edo Méx' y 'Cambray Rosa DF.' 'Cambray Rosa Morelos' y 'Mondial' fueron los más distantes (0.62).

Un análisis combinado con marcadores moleculares y morfológicos permitió la formación de cuatro grupos a un coeficiente de 0.72 (Figura 4), con un rango de similitudes entre 0.58 a 0.91 . 'Cambray Rosa Morelos' formó el primer grupo y fue el más divergente del resto de los cultivares. 'Criolla Edo. Méx', 'Papa chica,' 'Montserrat' y 'Tollocan' formaron el segundo. 'Mondial' formó el tercer grupo. El cuarto grupo por 'Gigant', 'Alfa,' 'Armada, 'Fianna, 'Cambray Blanca Edo. Méx', 'Cambray Rosa DF, 'Criolla Blanca Puebla', 'Mochis' y 'Atlantic'. Alta similitud se observó entre 'Armada' y 'Fianna,' 'Armada' y 'Alfa,' 'Cambray Rosa DF' y 'Criolla Blanca Puebla. Los menos relacionados fueron 'Cambray Rosa Morelos' y 'Mondial', 'Papa Chica' y 'Mondial, 'Fianna' y 'Cambray Rosa Morelos'.

Según la genealogía indicada en el Cuadro 1, 'Fianna’ y 'Gigant' comparten alguna porción de genoma del progenitor AM-66-42, sin embargo no se detectó una marcada relación entre ellos al utilizar marcadores moleculares o morfológicos. Los caracteres morfológicos cualitativos, los combinados morfológicos (cualitativos más cuantitativos) y los combinados moleculares (RAPD más ISSR) más morfológicos agruparon a las dos variedades en un mismo grupo, pero distantes.

La prueba de Mantel mostró baja correlación entre caracteres cualitativos y cuantitativos (0.317) (Cuadro 4). Ello quedó demostrado con el hecho de que cultivares diferentes fueron asignados a grupos diferentes al usar uno $\mathrm{u}$ otro tipo de marcador. 'Fianna', 'Armada' y 'Alfa' formaron grupos diferentes al usar caracteres cuantitativos, mientras que los mismos cultivares se agruparon igual cuando se usaron caracteres cualitativos (Figuras 1A y 1B). Se obtuvo una correlación alta entre matrices de caracteres morfológicos combinados y los respectivos caracteres cuantitativos y cualitativos por separado (Cuadro 4). El análisis de caracteres morfológicos combinados resultó en un dendrograma semejante a los obtenidos con caracteres cualitativos 
A)

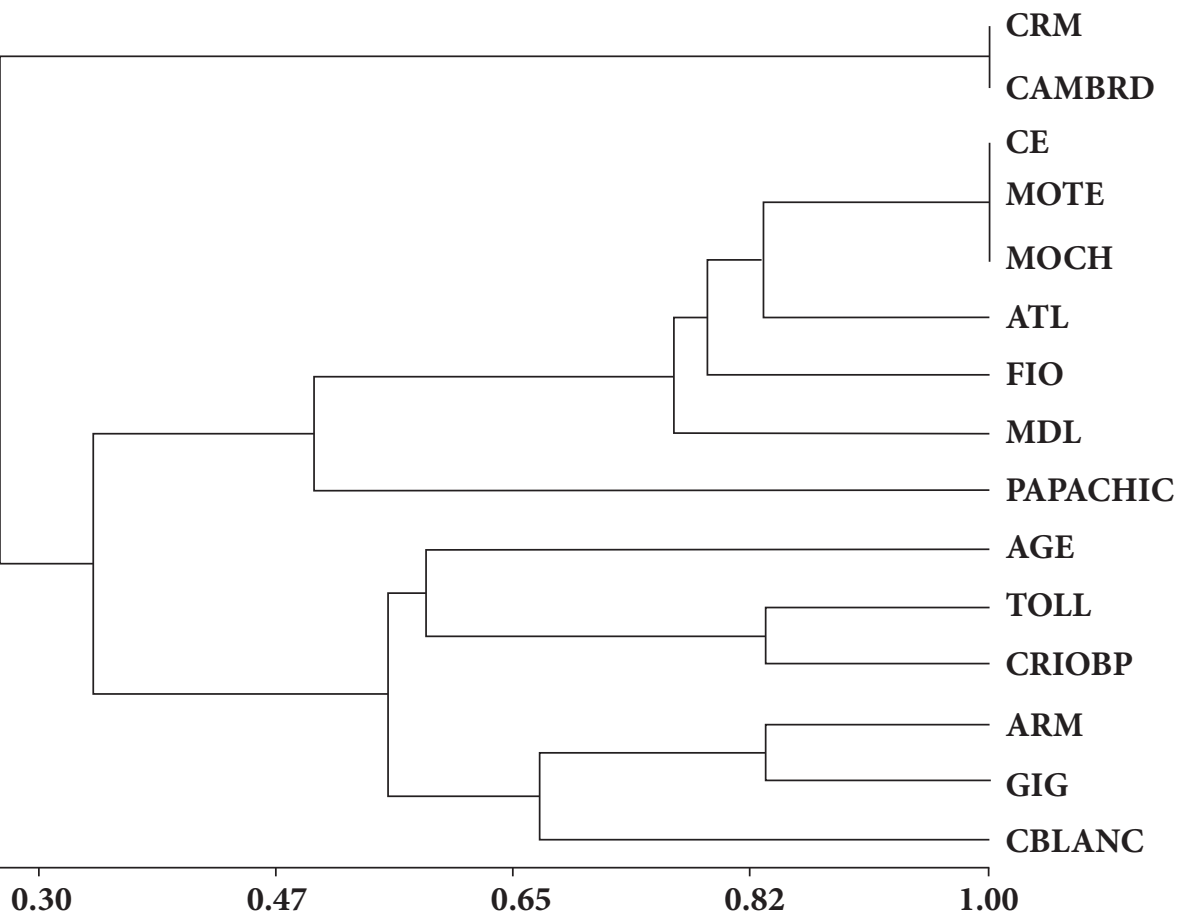

Coeficiente SM

B)

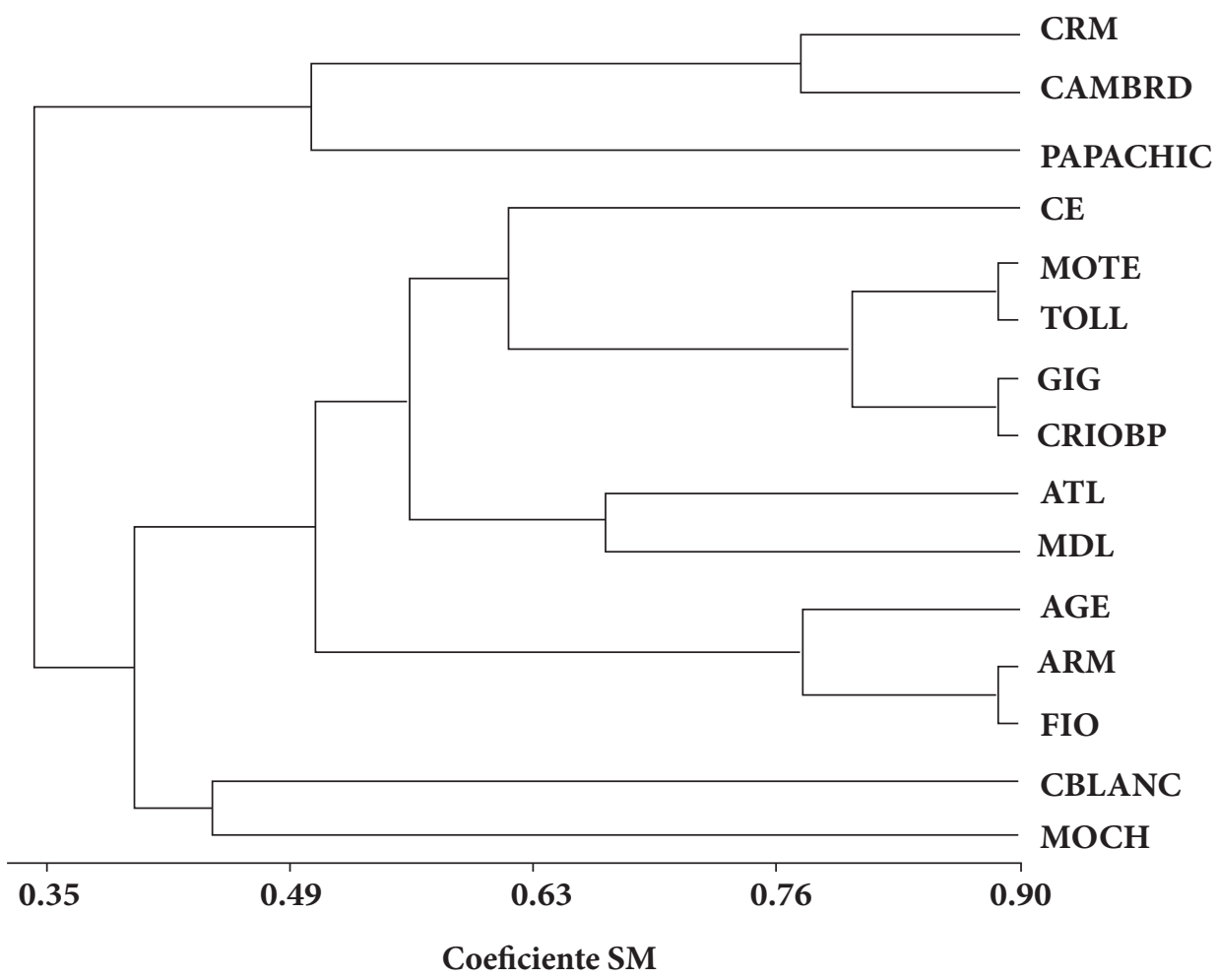

Figura 1. Dendrogramas generados a partir de datos cuantitativos (A), cualitativos (B) mediante el coeficiente de concordancia simple (SM) y el método de agrupamiento UPGMA, para 15 cultivares de papa. 


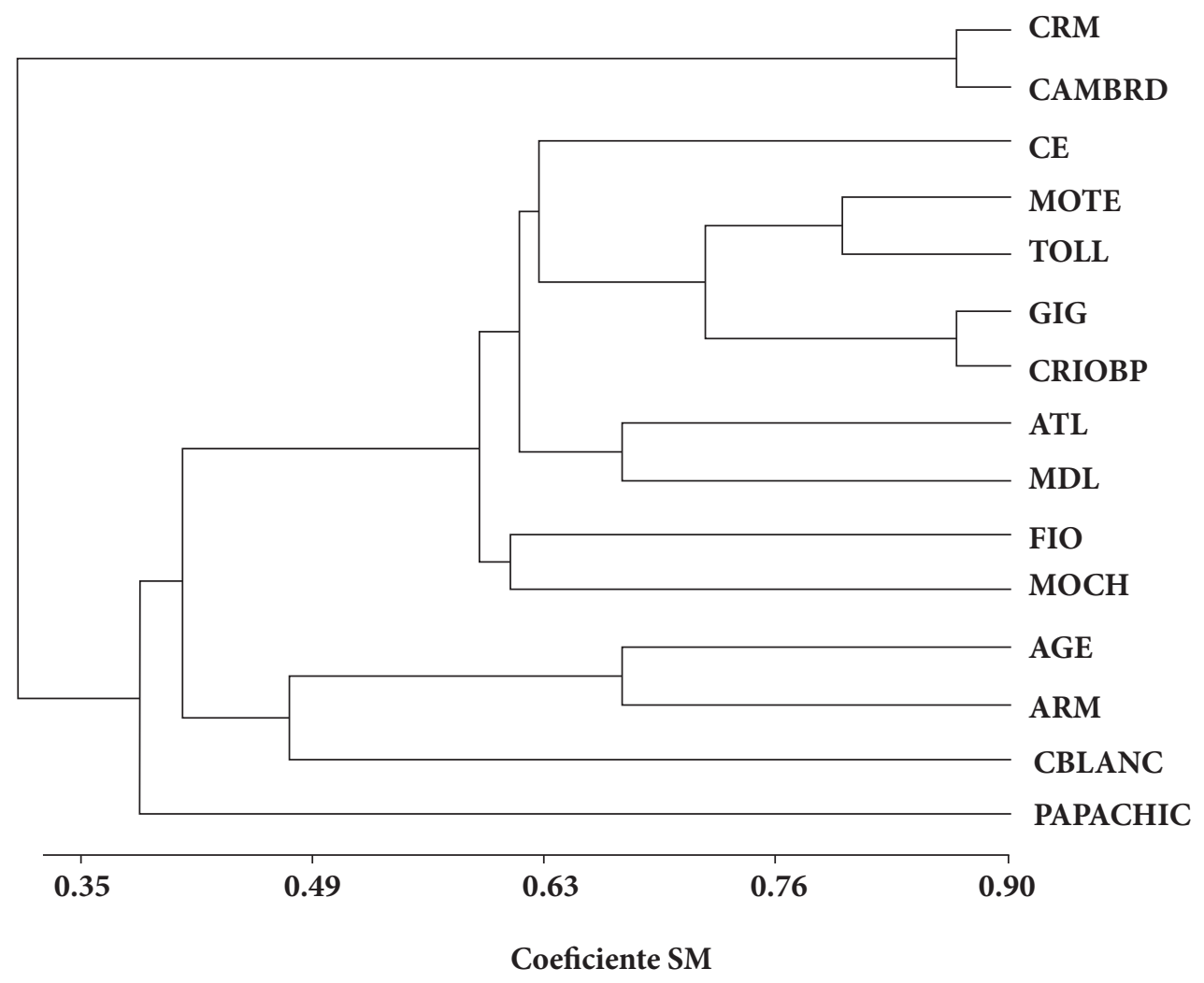

Figura 2. Dendrogramas generados a partir de datos cualitativos y cuantitativos combinados mediante el coeficiente de concordancia simple (SM) y el método de agrupamiento UPGMA, para 15 cultivares de papa.

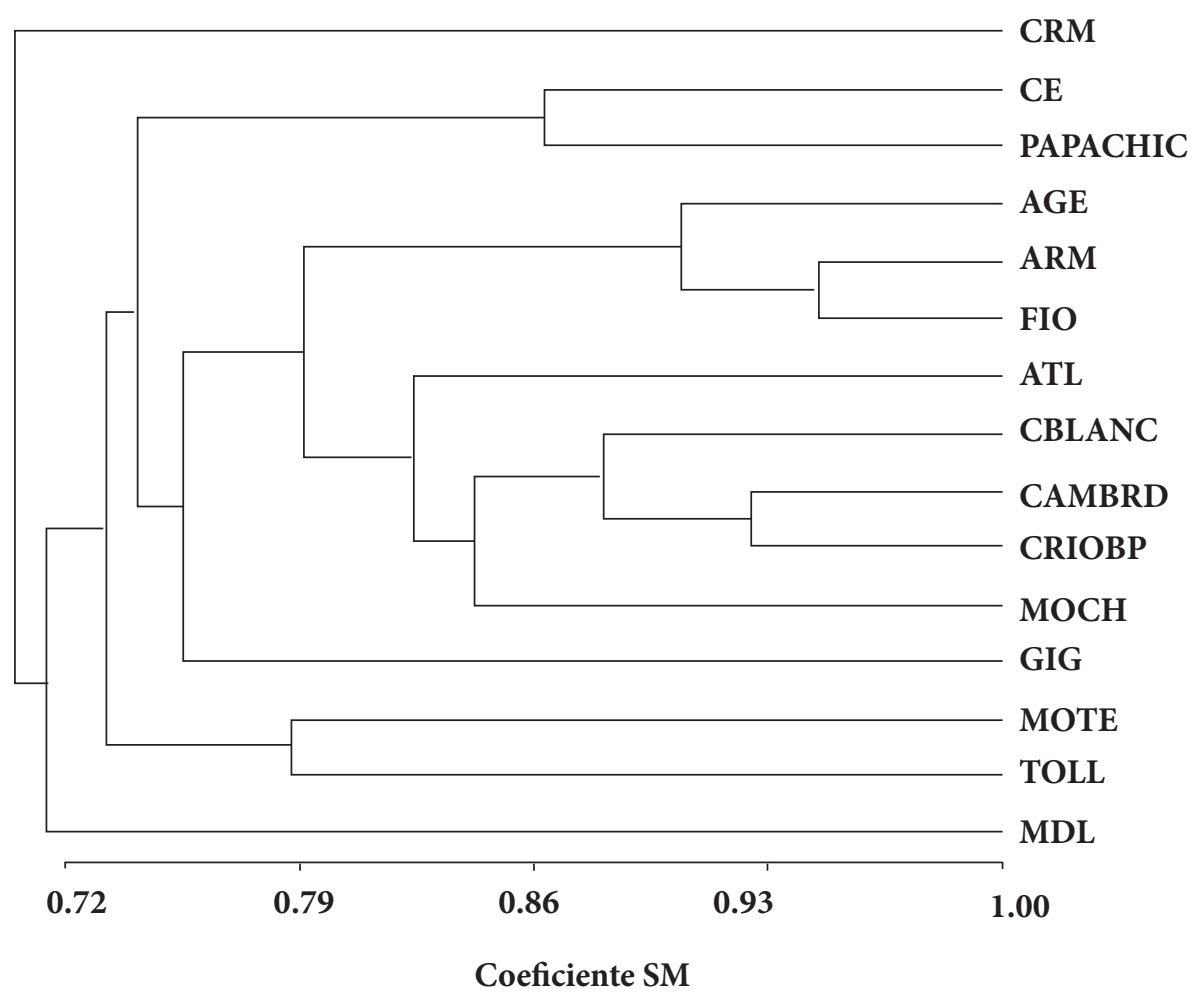

Figura 3. Dendrograma generado a partir de datos de marcadores moleculares consenso RAPD + ISSR mediante el coeficiente de concordancia simple (SM) y el método de agrupamiento UPGMA, para 15 cultivares de papa. 


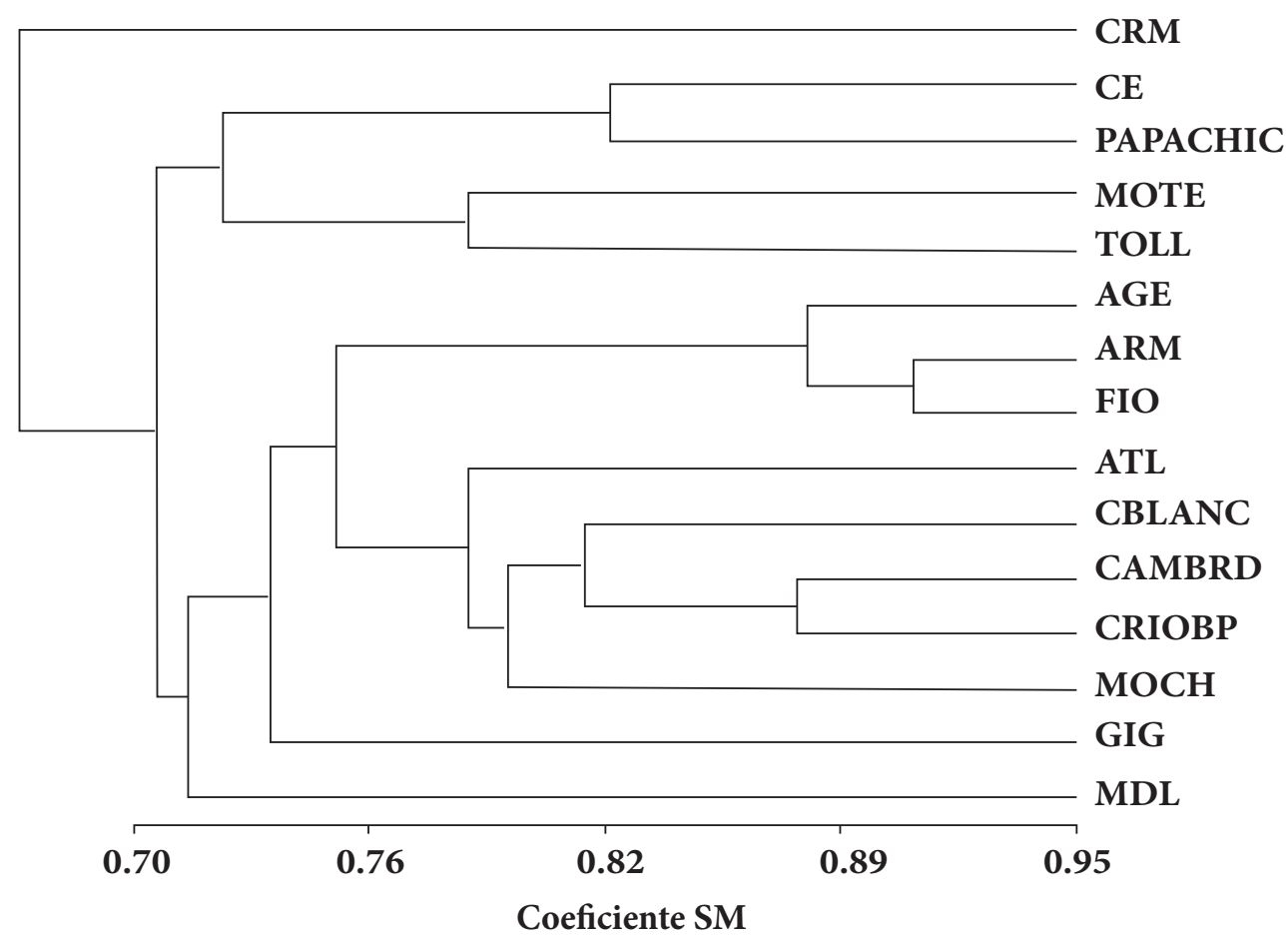

Figura 4. Dendrograma generado a partir de datos combinados de marcadores morfológicos y moleculares mediante el coeficiente de concordancia simple (SM) y el método de agrupamiento UPGMA, para 15 cultivares de papa.

o cuantitativos por separado. 'Atlantic' y 'Mondial' fueron agrupados en el mismo subgrupo, como se observa en el dendrograma generado al analizar caracteres cualitativos (Figura 1B). 'Cambray Rosa Morelos', 'Cambray Rosa DF' y 'Papa Chica' se agruparon también como se muestra en el dendrograma para caracteres cuantitativos (Figura 1A).

La prueba de Mantel mostró baja y negativa correlación entre matrices generadas con marcadores morfológicos y moleculares (-0.04) (Cuadro 4). Esto puede deberse a que los dos análisis evalúan diferentes caracteres. Los cultivares que se agruparon juntos al usar marcadores morfológicos, fueron colocados en grupos diferentes al usar marcadores moleculares, lo que sugiere que ellos son fenotípicamente similares pero genotípicamente diferentes, lo que muestra plasticidad. 'Fianna' se agrupó con 'Mochis' con marcadores morfológicos. A la inversa, 'Fianna' se agrupó con 'Alfa' y 'Armada' con marcadores moleculares. 'Cambray Rosa Morelos' agrupó junto con 'Cambray Rosa DF' al usar marcadores morfológicos; sin embargo, los dos cultivares fueron colocados en grupos diferentes al usar marcadores moleculares (Figuras 2 y 3 ). Cultivares que fueron genotípicamente similares pero morfológicamente diferentes incluyeron a 'Fianna' y 'Armada, 'Criolla Edo Méx', 'Papa Chica' y 'Atlantic', 'Cambray Rosa DF, 'Cambray Blanca Edo Méx', 'Criolla Blanca Puebla' y 'Mochis'. Lo anterior indica que no hubo correspondencia entre los marcadores morfológicos y moleculares evaluados, lo cual tiene sentido porque los caracteres evaluados con ambos tipos de marcadores son diferentes, pero complementarios.

Los marcadores moleculares RAPD e ISSR son abundantes y amplifican una amplia región del genoma, y por tal razón estos marcadores son capaces de discriminar entre individuos altamente relacionados. La prueba de Mantel para asociación entre las matrices derivadas de datos moleculares y morfológicos indicó una pobre correlación, lo que demuestra que los dos métodos discriminan de manera diferente entre los genotipos. Varios investigadores han reportado baja correlación entre marcadores morfológicos y moleculares para varios cultivos (Karuri et al., 2010; Koehler-Santos et al., 2003; Ferriol et al., 2004), y al respecto sugieren que ello puede deberse a la naturaleza independiente de las variaciones morfológicas y moleculares.

La baja correlación puede deberse también al hecho de que una gran porción de variación detectada por los marcadores moleculares es no-adaptativa, y por tanto no está sujeta a la selección natural o artificial como sucede con los caracteres morfológicos (Vieira et al., 2007). La prueba de Mantel mostró una correlación alta y positiva entre matrices combinadas de marcadores morfológicos y moleculares vs. marcadores moleculares (0.957) (Cuadro 4). Combinar datos morfológicos y moleculares agrupó a los cultivares según un patrón semejante al que resulta de usar sólo marcadores moleculares (Figuras 3 y 4 ). Probablemente los marcadores moleculares tienen mayor influencia sobre el análisis combinado debido al mayor 
Cuadro 4. Prueba de Mantel entre marcadores y valores de r.

\begin{tabular}{clc}
\hline Núm. & \multicolumn{1}{c}{ Matriz de correlaciones } & $\mathrm{r}$ \\
\hline 1 & Cualitativos vs. Cuantitativos & $0.32^{\star}$ \\
2 & Combinados Cuantitativos y Cualitativos vs. Cualitativos & $0.82^{\star}$ \\
3 & Combinados Cuantitativos y Cualitativos vs. Cuantitativos & $0.80^{\star}$ \\
4 & Combinados Morfológicos y Moleculares vs. Cualitativos & $0.33^{\star}$ \\
5 & Combinados Morfológicos y Moleculares vs. Cuantitativos & $0.07^{\star}$ \\
6 & Combinados Morfológicos y Moleculares vs. Combinados Cuantitativos y Cualitativos & $0.25^{\star}$ \\
7 & Combinados Morfológicos y Moleculares vs. Moleculares & $0.96^{*}$ \\
8 & Combinados Cuantitativos y Cualitativos vs. Moleculares & $-0.04^{*}$ \\
\hline
\end{tabular}

${ }^{*}$ Significativo con $\alpha=0.05$.

número de loci incluidos en el análisis.

\section{Caracterización morfológica mediante escalado mul- tidimensional}

El escalado multidimensional obtenido mediante caracteres cuantitativos dividió a los cultivares de papa en cinco grupos (Figura 5A). Cultivares que muestran hojas grandes, con gran longitud y ancho de hojas secundarias agruparon juntos ('Cambray Blanca Edo Méx', 'Armada', 'Gigant', 'Criolla Blanca Puebla' y 'Tollocan'). Cultivares con hojas de tamaño mediano y longitud y anchos medianos de hojas secundarias, formaron un segundo grupo ('Fianna', 'Mondial', 'Mochis, 'Montserrat', 'Criolla Edo Méx' y 'Papa chica'). 'Atlantic' formó su propio grupo con base en hojas de tamaño pequeño, de dimensiones medianas en longitud y ancho de hojas secundarias. 'Alfa' también formó un grupo separado con base en la característica hoja terminal de tamaño pequeño. 'Cambray Rosa Morelos' y 'Cambray Rosa DF' se agruparon juntas por tener hojas de tamaño pequeño y hojas secundarias también pequeñas.

Los caracteres cualitativos aglutinaron a los cultivares en cuatro grupos (Figura 5B). 'Cambray Blanca Edo Méx’ formó un grupo separado por la presencia moderada de hojas secundarias y hoja terminal pequeña. Los cultivares con tubérculos rojos y redondos y que no mostraron coalescencia de hojas terminales con hojas primarias agruparon juntos ('Papa Chica', 'Criolla Edo Méx', y 'Cambray Rosa DF'). 'Cambray Rosa Morelos’ agrupó aparte. El resto de los cultivares formó otro grupo por sus hojas terminales de tamaño medio, estructura abierta de hoja y tubérculos de cubierta o piel color blanco.

La gráfica generada al usar la combinación de datos de marcadores morfológicos dividió a los cultivares en cuatro grupos (Figura 5C). Se formaron grupos semejantes a los obtenidos con sólo marcadores cualitativos, con excepción de un cuarto grupo integrado por 'Atlantic', 'Mochis' y 'Papa Chica' cuyas hojas secundarias son de longitud y ancho medianos.

Los dendrogramas y el escalado multidimensional agruparon a los cultivares en algunos casos en forma diferente cuando se usaron marcadores morfológicos. Algunos cultivares mantuvieron su agrupamiento al usar ambos métodos. Esto se observó también en tres grupos separados formados por 'Cambray Rosa Morelos, 'Gigant' y 'Fianna, 'Armada' y 'Alfa', al usar marcadores moleculares.

Utilizar datos cualitativos o la combinación de marcadores morfológicos permitió agrupar a 'Fianna' y 'Gigant' en un mismo grupo. En general, el escalado multidimensional permitió mejores agrupamientos que el análisis de conglomerados.

\section{CONCLUSIONES}

Los marcadores morfológicos y moleculares detectaron diversidad genética entre los cultivares. Si bien ambos marcadores diferenciaron los cultivares de papa, los moleculares discriminaron completamente a los 15 cultivares analizados, mientras que los morfológicos fueron incapaces de discriminarlos en su totalidad. Para que los programas de mejoramiento sean eficientes es importante seleccionar líneas con base en la diversidad genética de parámetros agromorfológicos, asistidos con marcadores moleculares.

\section{AGRADECIMIENTOS}

El primer autor le agradece al Dr. Héctor Lozoya Saldaña del Departamento de Fitotecnia de la Universidad Autónoma Chapingo, México por proporcionar algunos materiales de papa. 

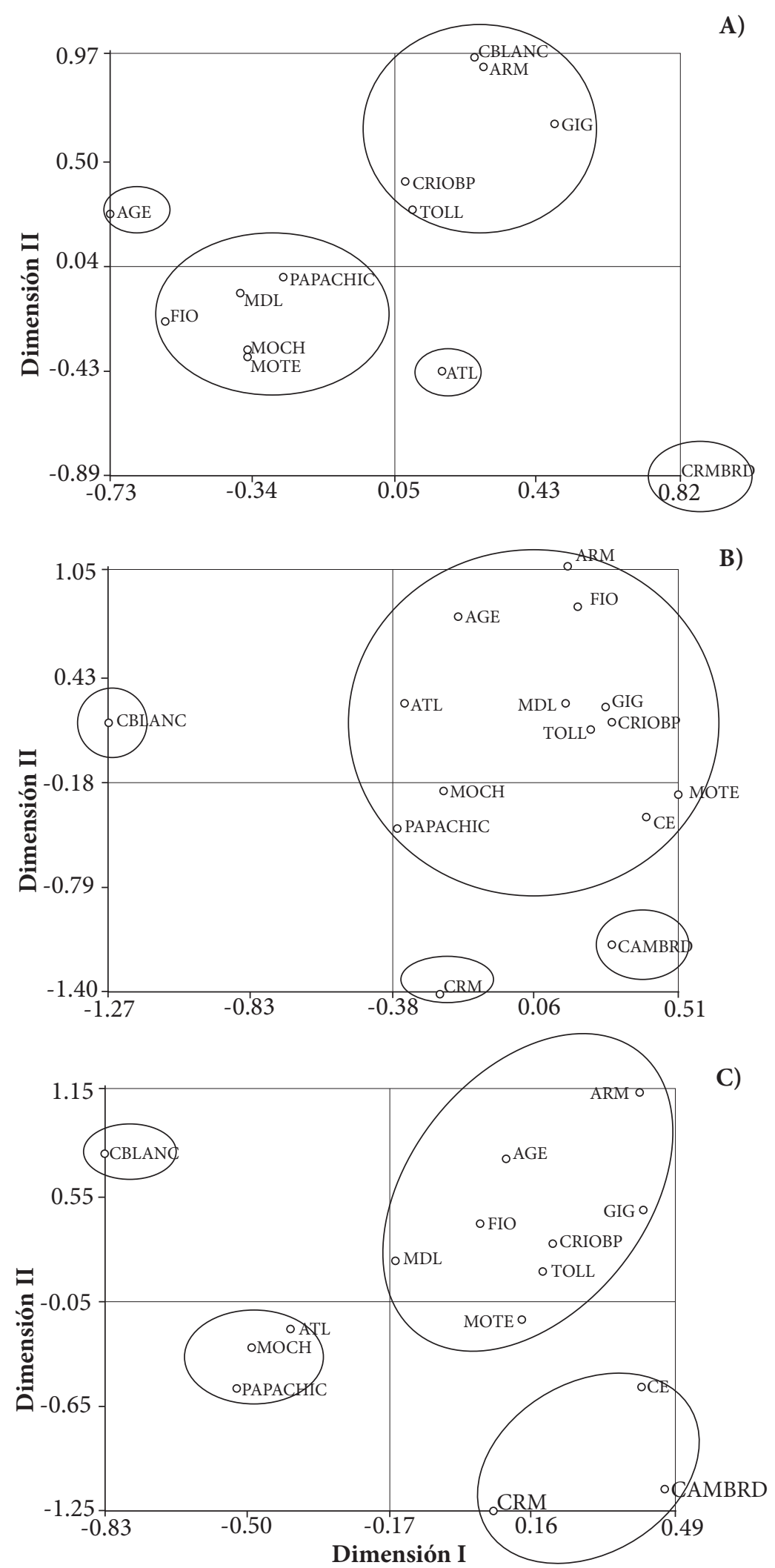

Figura 5. Gráfica en 2D generada mediante análisis de escalado multidimensional con datos cuantitativos (A), datos cualitativos (B) y datos combinados cuantitativos y cualitativos (C), de 15 cultivares de papa. 


\section{BIBLIOGRAFÍA}

Ahmadizadeh M, H Felenji (2011) Evaluating diversity among potato cultivars using agro-morphological and yield components in fall cultivation of Jiroft area. Amer. Eurasian J. Agric. Environ. Sci.11:655-662.

Arslanoglu F, S Aytac, E K Oner (2011) Morphological characterization of the local potato (Solanum tuberosum L.) genotypes collected from the Eastern Black Sea region of Turkey. African J. Biotechnol.10:922-932.

Becerra V V, C M Paredes (2000) Uso de marcadores bioquímicos y moleculares en estudios de diversidad genética. Agric. Téc. (Chile) 60:270-281.

Chimote V P, D Pattanayak, P C Naik (2007) Molecular and morphological divergence studies in Indian potato varieties. Indian J. Biotechnol. 6:216-223.

Dellaporta S L, J Wood, J B Hicks (1983) A plant DNA mini preparation: Version II. Plant Mol. Biol. Rep. 1:19-21.

Ferriol M, B Pico, D Fernandez, F Nuez (2004) Molecular diversity of germplasm collection of squash (Cucurbita moschata) determined by SRAP and AFLP markers. Crop Sci. 44:653-664.

Hawkes J G (1990) The potato: evolution, biodiversity and genetic resources. Belhaven Press. Washington DC. pp:1-50.

Hijmans R J, M Jacobs, J B Bamberg, D M Spooner (2003) Frost tolerance in wild potato species: assessing the predictivity of taxonomic, geographic, and ecological factors. Euphytica 130:47-59.

Instituto Nacional de Investigaciones Agrícolas (1981) Guía para la Asistencia Técnica Agrícola. Área de Influencia del CAEVAMEX. SAG. 79 p.

Karuri H W, E M Ateka, R Amata, A B Nyende, A W T Muigai, E Mwasame, S T Gichuki (2010) Evaluating diversity among Kenyan sweet potato genotypes using morphological and SSR markers. Int. J. Agric. Biol. 12:33-38.

Koehler-Santos P, A L Dornelles, L B de Freitas (2003) Characterization of mandarin citrus germplasm from southern Brazil by morphological and molecular analysis. Pesq. Agropec. Bras. 38:797-806.

Lara-Cabrera S I, D M Spooner (2004) Taxonomy of North and Central American diploid wild potato (Solanum sect. Petota) species: AFLP data. Plant Syst. Evol. 248: 129-142.

Mantel N (1967) The detection of disease clustering and a generalized regression approach. Cancer Res. 27:209-220.

Orona-Castro F, V Pecina-Quintero, A Rocha-Peña, M A Cadena-Hinojosa, O O Martínez de la Vega, I H Almeyda-León (2006) Caracterización molecular de genotipos comerciales y elite de papa. Agric. Téc. Méx. 2:171-180.
Rocha E A, L V Paiva, H H de Carvalho, C T Guimaraes (2010) Molecular characterization and genetic diversity of potato cultivars using SSR and RAPD markers. Crop Breed. Appl. Biotechnol. 10:204-210.

Rohlf F J (2000) Programme NTSYS-pc: numerical taxonomy and multivariate analysis system: version 2.1. Exeter Software, New York.

SAGARPA, Secretaría de Agricultura, Ganadería, Desarrollo Rural, Pesca y Alimentación (2011) Servicio de información y estadística agroalimentaria y pesquera. Cultivo de papa. México, D. F. Disponible en Siap.gob.mx. (Septiembre 2012).

Sneath P H, R R Sokal (1973) Numerical taxonomy. The Principles and Practice of Numerical Classification. W.H. Freeman and Company. San Francisco, Calif. 189 p.

Solís J S, D M Ulloa, L A Rodríguez (2007) Molecular description and similarity relationships among native germplasm potatoes (Solanum tuberosum spp) using morphological data and AFLP markers. Electr. J. Biotechnol. 10:11-20.

Spooner D M, S Lara-Cabrera (2001) Sistemática molecular y evolución de plantas cultivadas. In: H M Hernández, A García-Aldrete, F Álvarez y M Ulloa (eds). Enfoques Contemporáneos para el Estudio de la Biodiversidad. Instituto de Biología, UNAM. Fondo de Cultura Económica. México DF. pp:57-114.

Spooner D M, R G van den Berg, G Bryan, A del Rio (2003) Species concepts and relationships in wild and cultivated potatoes. Acta Hort. 619:63-75.

Spooner D M, R G van den Berg, A Rodriguez, J Bamberg, R J Hijmans, S Lara-Cabrera (2004) Wild potatoes (Solanum section Petota); of North and Central America. Syst. Bot. Monogr. 68:1-209.

Spooner D M, J Núñez, G Trujillo, M R Herrera, F Guzmán, M Ghislain (2007) Extensive simple sequence repeat genotyping of potato landraces supports a major reevaluation of their gene pool structure and classification. PNAS 104:19398-19403.

Spooner D, F Rodríguez, Z Polgár, H E Ballard, S H Jansky (2008) Genomic origins of potato polyploids: GBSSI gene sequencing data. The Plant Genome. A Suppl. to Crop Sci. 48:S27-S36.

UPOV, International Union for the Protection of New Varieties of Plants (2004) Potato Guidelines for the Conduct of Tests for Distinctness, Uniformity and Stability for Potato (Solanum tuberosum L.). Document TG/88/6:1-24.

Vieira E, F Carvalho, I Bertan, M Kopp, P Zimmer, G Benin, J da Silva, I Hartwig, G Malone, A de Oliviera (2007) Association between genetic distances in wheat (Triticum aestivum L.) as estimated by AFLP and morphological markers. Gen. Mol. Biol. 30:392-399.

Yasmin S, M S Islam, M Kondoker, M Nasiruddin, S Alam (2006) Molecular characterization of potato germplasm by Random Amplified Polymorphic DNA markers. Biotechnol. 5:27-31. 